PROCEEDINGS OF THE

AMERICAN MATHEMATICAL SOCIETY

Volume 139, Number 5, May 2011, Pages 1777-1785

S 0002-9939(2010)10630-5

Article electronically published on October 20, 2010

\title{
A COMPARISON PRINCIPLE FOR HAMILTON-JACOBI EQUATIONS WITH DISCONTINUOUS HAMILTONIANS
}

\author{
YOSHIKAZU GIGA, PRZEMYSŁAW GÓRKA, AND PIOTR RYBKA
}

(Communicated by Matthew J. Gursky)

\begin{abstract}
We show a comparison principle for viscosity super- and subsolutions to Hamilton-Jacobi equations with discontinuous Hamiltonians. The key point is that the Hamiltonian depends upon $u$ and has a special structure. The supersolution must enjoy some additional regularity.
\end{abstract}

\section{INTRODUCTION}

The purpose of this paper is to give a simple proof of a comparison principle for bounded, uniformly continuous sub- and supersolution solutions to the HamiltonJacobi equation

$$
d_{t}+H\left(t, x, d, d_{x}\right)=0 \quad \text { in }(0, T) \times \mathbb{R}
$$

when the Hamiltonian $H$ is discontinuous and depends in a non-trivial way on $d$. It is well-known that, in general, if $H$ is discontinuous in $x$, then the comparison principle may fail.

Here, we assume a special structure of $H$ and its line of discontinuity. It comes from the singular curvature flow, considered in 8]. Namely, the equation studied there leads to the following form of $H$ :

$$
H(t, x, u, p)= \begin{cases}-\sigma\left(t, r^{*}(t), u\right) m(p) & \text { if }|x|<r_{0}(t) \\ -\sigma(t, x, u) m(p) & \text { if }|x| \geq r_{0}(t) .\end{cases}
$$

Here, we explain the assumption starting from the line of discontinuity.

(R1) $r_{0}$ and $r^{*}$ belong to $C^{0}([0, T])$ and $r^{*}(t)>r_{0}(t)$ for all $t \in[0, T]$. In addition, the set $\left\{\left(t, r_{0}(t)\right): t \in[0, T]\right\}$ is a Lipschitz curve.

Received by the editors February 19, 2010 and, in revised form, May 23, 2010.

2010 Mathematics Subject Classification. Primary 49L25.

Key words and phrases. Hamilton-Jacobi equation, viscosity solutions, discontinuous Hamiltonian.

The work of the first author was partly supported by a Grant-in-Aid for Exploratory Research (20654017) and a Grant-in-Aid for Scientific Research (S) (21224001) from the Japan Society for the Promotion of Science.

The second and third authors were partly supported by the Polish Ministry of Science grant N N2101 268935. The second author also enjoyed partial support from Fondecyt 3100019.

The third author thanks Hokkaido University for its hospitality, as part of the research was performed while he was visiting the university. His work has been partially supported also by FONDAP-Chile.

(C)2010 American Mathematical Society Reverts to public domain 28 years from publication 
Let us remark that $r_{0}$ need not be Lipschitz continuous as a function of $t$ as in the case of $r_{0}(t)=\sqrt{t}+1$. The set in question is a subset of a parabola.

The conditions we present are not optimal, but they are simple enough and permit us to present the main argument. We have to specify restrictions on the other components. We assume that $\sigma \in C^{1}$ is bounded, even as a function of $x$ or $u$, while other arguments are fixed. It is also increasing with respect to $x$ as well as $u$, provided that $x, u>0$. In addition,

$$
0<\sigma_{u}(t, x, u) \leq M
$$

and $m$ is a positive convex function with linear growth at infinity. In the present paper, however, no conditions on $m$ are necessary except for continuity.

In [8, Theorem 4.3] we showed a comparison principle for special bounded, even, Lipschitz continuous sub- and supersolution solutions to (1.1). We required in [8] that the supersolution be increasing on $[0,+\infty)$, while the subsolution be constant over $[0, a(t)]$ for $a(t) \geq r_{0}(t)$ for all $t \in[0, T]$.

Here, we prove the Comparison Principle (see Theorem 2.7) without these structural restrictions on sub- and supersolution solutions; however, we impose moderate regularity assumptions. Before explaining our method, we will comment on the available literature.

Let us mention that while the notion of semicontinuous super- and subsolutions for discontinuous Hamiltonians is well-defined (see [1], 9] [10]), the authors frequently assume in the statements of their Comparison Principles that either the supersolution of the subsolution is at least Lipschitz continuous [2, 3], 4], 5], 6], 11.

There are various kinds of motivation for studying problems like (1.1). One stems from image analysis, like the 'shape-from-shading' problem [4, 11, and another is from flame propagation or etching 3 , or from game theory [6]. In those papers (1.1) is a general form of the eikonal equation and $H$ does not depend upon $u$. For us (1.1) is a result of degeneration of a second order parabolic problem (see [8]) where the dependence of $H$ upon $u$ is essential.

There is a spectrum of assumptions on admissible discontinuities with respect to $x$ and $t$. Jump discontinuities across Lipschitz hypersurfaces are quite common [3], [5], 6], 11]. We may add that sometimes the authors admit triple junctions of the discontinuity set; see [5. In [4 jump discontinuities are admitted along a set of vertical and horizontal intervals. The most general situation is considered in [2], where the authors must use tools from measure theory and consider a slightly different notion of solution.

Our comparison principle does not require any special condition on the kind of dependence of $H$ upon $p$ except continuity. On the other hand, 2, 4, 5 need coercitivity, while the authors of [2], 11] assume convexity in $p$. Sometimes other conditions are used, such as 1-homogeneity with respect to $p$ (see [6]) or linear growth at infinity (see [3]).

We deal, however, with the graph over the real line, so we have to control the behavior of supersolutions at infinity. For this purpose we introduce a convenient technical notion of supersolution at infinity. We also find it convenient to work with strict supersolution, but understood differently than in [11.

Our method of proof of Theorem 2.7 is based upon the idea of shifting the supersolution $v$ away from the discontinuity of $H$ so that the shifted $v$ becomes a strict supersolution. We also regularize $H$ in a proper manner, so that the strict 
supersolutions remain supersolutions and subsolutions remain subsolutions as well. This permits us to use classical results and deduce our claim.

\section{THE COMPARISON PRINCIPLE}

We first recall from [8, Definition 1] (see [1, 9] and more recently from [4] and also [7]) the notion of a sub-/supersolution to (1.1) in case of a discontinuous Hamiltonian.

Definition 2.1. (a) We shall say that a bounded, uniformly continuous function $u:(0, T) \times \mathbb{R} \rightarrow \mathbb{R}$ is a viscosity subsolution of (1.1) provided that for all $C^{1}$ functions $\varphi:(0, T) \times \mathbb{R} \rightarrow \mathbb{R}$ such that $u-\varphi$ has a local maximum at $\left(t_{0}, x_{0}\right)$, then

$$
\varphi_{t}\left(t_{0}, x_{0}\right)+H_{*}\left(t_{0}, x_{0}, u\left(t_{0}, x_{0}\right), \varphi_{x}\left(t_{0}, x_{0}\right)\right) \leq 0 .
$$

(b) We shall say that a bounded, uniformly continuous function $v:(0, T) \times \mathbb{R} \rightarrow \mathbb{R}$ is a viscosity supersolution of (1.1) if for all $C^{1}$ functions $\varphi:(0, T) \times \mathbb{R} \rightarrow \mathbb{R}$ such that $v-\varphi$ has a local minimum at $\left(t_{0}, x_{0}\right)$, then

$$
\varphi_{t}\left(t_{0}, x_{0}\right)+H^{*}\left(t_{0}, x_{0}, v\left(t_{0}, x_{0}\right), \varphi_{x}\left(t_{0}, x_{0}\right)\right) \geq 0 .
$$

(c) We shall say that a bounded, uniformly continuous function $d:(0, T) \times \mathbb{R} \rightarrow \mathbb{R}$ is a viscosity solution of (1.1) provided that it is a viscosity subsolution as well as a viscosity supersolution of (1.1).

For the sake of self-consistency we briefly recall the definitions of an upper semicontinuous envelope, $H^{*}$, and a lower semicontinuous envelope, $H_{*}$, for a locally bounded function $H:(0, T) \times \mathbb{R}^{3} \rightarrow \mathbb{R}$. Namely, we set

$$
H_{*}(z)=\liminf _{\zeta \rightarrow z} H(\zeta), \quad H^{*}(z)=\limsup _{\zeta \rightarrow z} H(\zeta) .
$$

We notice that Definition 2.1 is in the line of notion of sub-(super-)solution introduced by [1, 9] and more recently by [4] for discontinuous Hamiltonians.

We shall describe our assumptions on $H$ which slightly generalize formula (1.2) above.

We begin with continuity requirements:

(H1) Hamiltonian $H$ is lower semicontinuous in $[0, T] \times \mathbb{R} \times \mathbb{R} \times \mathbb{R}$.

(H2) $H$ is continuous away from $\Gamma \times \mathbb{R}^{2}$, where $\Gamma=\left\{\left(t, \pm r_{0}(t)\right): t \in[0, T]\right\}$ and has a jump discontinuity at $\Gamma \times \mathbb{R}^{2}$.

(H3) $H^{*}$ is continuous in $G \times \mathbb{R}^{2}$, where $G=\left\{(t, x):|x| \geq r_{0}(t)\right\}$, while $H_{*}$ is continuous on the closure of $(([0, T] \times \mathbb{R}) \backslash G) \times \mathbb{R}^{2}$.

Remark 2.2. Essentially it is possible to express (H3) just in terms of $H$, but we do not find it elegant. We could say that the set $(([0, T] \times \mathbb{R}) \backslash \Gamma) \times \mathbb{R}^{2}$ has two connected components $\mathcal{U}_{1}$ and $\mathcal{U}_{2}$. The function $H$ is continuous on each of the components and can be continuously extended from $\mathcal{U}_{i}$ onto its closure $\overline{\mathcal{U}}_{i}, i=1,2$.

Symmetry of $H$ is just for the sake of simplicity. That is, we impose:

(H4) For any $\epsilon_{1}, \epsilon_{2}$ in $\{-1,1\}$ we have $H\left(t, \epsilon_{1} x, u, \epsilon_{2} p\right)=H(t, x, u, p)$.

Monotonicity of our Hamiltonian is crucial for our argument. We shall frequently use the following condition:

(H5) The Hamiltonian $H$ is strictly increasing with respect to $u$; i.e. there is a positive constant $h_{0}$ such that the following inequality holds for all $u_{2}, u_{1}, x, t$ and $p$ :

$$
H\left(t, x, u_{2}, p\right)-H\left(t, x, u_{1}, p\right) \geq h_{0}\left(u_{2}-u_{1}\right) .
$$


(H6) For all $t, u$ and $p$, the function $x \mapsto H(t, x, u, p)$ is decreasing for $x>r_{0}(t)$; moreover, $H(t, x, u, p)=H\left(t, r^{*}(t), u, p\right)$ for $x \in\left[-r_{0}(t), r_{0}(t)\right]$.

Remark 2.3. It is possible to convert $H$ given by (1.2) into one satisfying (2.1) by means of the following change of variables $v=e^{t \lambda} u$, where $\lambda=-2 M$ and $M$ is the constant appearing in (1.3). Nonetheless, even the transformed Hamiltonian, $H_{\text {new }}$, will have a jump in $(t, x)$ at $\left(t, \pm r_{0}(t)\right)$. It has the following form:

$$
H_{\text {new }}(t, x, v, p)=2 M v+e^{-2 M t} H\left(t, x, e^{2 M t} v, e^{2 M t} p\right) .
$$

Interestingly, property (2.1) is inherited by $H^{*}$ and $H_{*}$.

Corollary 2.4. If $H$ satisfies (2.1), then so do $H^{*}$ and $H_{*}$ with the same constant $h_{0}$.

Proof. By the definition of $H^{*}(t, x, u, p)$ there is a sequence $\left(t_{n}, x_{n}, u_{n}^{1}, p_{n}\right)$ converging to $\left(t, x, u_{1}, p\right)$ such that

$$
\lim _{n \rightarrow \infty} H\left(t_{n}, x_{n}, u_{n}^{1}, p_{n}\right)=H^{*}\left(t, x, u_{1}, p\right) .
$$

By (2.1) we have

$$
H\left(t_{n}, x_{n}, u_{2}, p_{n}\right)-H\left(t_{n}, x_{n}, u_{n}^{1}, p_{n}\right) \geq h_{0}\left(u_{2}-u_{n}^{1}\right) .
$$

By definition of $H^{*}$ the inequality $H^{*}\left(t_{n}, x_{n}, u_{2}, p_{n}\right) \geq H\left(t_{n}, x_{n}, u_{2}, p_{n}\right)$ always holds. Since $H^{*}$ is upper semicontinuous we have

$$
\begin{aligned}
H^{*}\left(t, x, u_{2}, p\right)-H^{*}\left(t, x, u_{1}, p\right) & \geq \limsup _{n \rightarrow \infty} H\left(t_{n}, x_{n}, u_{2}, p_{n}\right)-\lim _{n \rightarrow \infty} H\left(t_{n}, x_{n}, u_{n}^{1}, p_{n}\right) \\
& \geq \lim _{n \rightarrow \infty} h_{0}\left(u_{2}-u_{n}^{1}\right)=h_{0}\left(u_{2}-u_{1}\right) .
\end{aligned}
$$

Hence, $H^{*}$ indeed satisfies (2.1).

In order to show (2.1) for $H_{*}$ we proceed in a similar way: we take a sequence $\left(t_{n}, x_{n}, u_{n}^{2}, p_{n}\right)$ converging to $\left(t, x, u_{2}, p\right)$ such that

$$
\lim _{n \rightarrow \infty} H\left(t_{n}, x_{n}, u_{n}^{2}, p_{n}\right)=H^{*}(t, x, u, p) .
$$

Subsequently, we apply the liminf to the inequality

$$
H\left(t_{n}, x_{n}, u_{n}^{2}, p_{n}\right)-H_{*}\left(t_{n}, x_{n}, u_{n}^{1}, p_{n}\right) \geq h_{0}\left(u_{n}^{2}-u_{1}\right) .
$$

Our claim follows.

In order to state and establish our result we need a technical device which is used to control the behavior of a supersolution at infinity. This is so because our region has no boundary. This requires another condition on the Hamiltonian:

(H7) $\lim _{\substack{|x| \rightarrow \infty \\ p \rightarrow 0}} H(t, x, u, p)=H^{\infty}(t, u)$, and the convergence is locally uniform with respect to $(t, u) \in[0, T] \times \mathbb{R}$. In particular, $H^{\infty}$ does not depend upon $p$.

In 8 ] we considered in fact piecewise $C^{1}$ solutions. We need them here as well. We also make precise what we shall call here a piecewise $C^{1}$ function in order to make the next notion more meaningful.

Definition 2.5. We shall say that a Lipschitz continuous function $w$ is a piecewise $C^{1}$-function (with discontinuity of the derivative along $\left\{|x|=r_{0}(t)\right\}$ ) provided that there are disjoint open sets, $\mathcal{U}_{i} \subset(0, T) \times \mathbb{R}, i=1, \ldots, N_{w}, N_{w} \in \mathbb{N}$, such that: (a) $[0, T] \times \mathbb{R}=\bigcup_{i=1}^{N_{w}} \overline{\mathcal{U}}_{i}$, (b) each $\mathcal{U}_{i}$ has Lipschitz boundary, (c) the set 
$\left\{t \in[0, T]:\left(t, r_{0}(t)\right),\left(t,-r_{0}(t)\right)\right\}$ is contained in $\bigcup_{i} \partial \mathcal{U}_{i},(\mathrm{~d})$ there exist two indexes $i_{0}$ and $j_{0}$ and a positive number $\mu_{0}$ such that

$$
(0, T) \times\left(-\infty,-\mu_{0}\right] \subset \overline{\mathcal{U}}_{i_{0}} \text { and }(0, T) \times\left[\mu_{0},+\infty\right) \subset \overline{\mathcal{U}}_{j_{0}},
$$

(e) $\left.w\right|_{\overline{\mathcal{U}}_{i}} \in C^{1}\left(\overline{\mathcal{U}}_{i}\right)$; i.e. the derivatives can be extended to $\overline{\mathcal{U}}_{i}$ as continuous functions.

Once we have imposed restrictions on the behavior of $H$ by requiring (H7), we can introduce another notion.

Definition 2.6. For $H$ satisfying (H7) we shall say that a piecewise $C^{1}$-function $w$ is a supersolution at infinity provided that $w$ is a supersolution and that the following limits exist and are uniform with respect to $t \in[0, T]$ :

$$
w_{t} \rightarrow w_{t}^{\infty}, \quad w \rightarrow w^{\infty}, \quad w_{x} \rightarrow 0 \quad \text { as }|x| \rightarrow \infty
$$

and

$$
w_{t}^{\infty}(t)+H^{\infty}\left(t, w^{\infty}(t)\right) \geq 0 .
$$

We shall call $w$ a strict supersolution at infinity if it is a supersolution at infinity and the inequality in (2.3) is strict.

Here is our main result. It is worth noticing that we do not impose on the Hamiltonian either coercitivity or convexity in $p$. In particular, the Hamiltonian given in (2.2) satisfies all our conditions provided that $\sigma$ in (1.2) converges uniformly, as $|x| \rightarrow \infty$, to some $\sigma^{\infty} \in C^{1}((0, T) \times \mathbb{R})$.

Theorem 2.7. Let us assume that a measurable function $H$ satisfies (R1) and (H1)-(H7) and that for $u, v \in B U C([0, T] \times \mathbb{R})$ the following conditions are valid:

(a) $v$ is a supersolution to (1.1), $u$ is a subsolution to (1.1) and $u(0, x) \leq v(0, x)$.

(b) $v$ is a piecewise $C^{1}$-function.

(c) $v$ is a supersolution of (1.1) at infinity.

Then, for all $t>0$,

$$
u(t, x) \leq v(t, x) .
$$

The above statement is rather long; however, the content is quite simple: we have to impose conditions permitting us to control the behavior of Hamiltonian $H$ and of super- and subsolutions at infinity. Moreover, we assume that the set of non-differentiability points of the supersolution is small and sets of discontinuities of $H$ and $v_{x}$ are 'aligned'.

We shall proceed in several stages: we will move the problem away from the jump discontinuity of $H$ by considering a "shifted supersolution". We also modify $H$ to make it a continuous function. Subsequently, we apply the classical results for continuous Hamiltonians.

In order to state our next observation it is convenient to introduce the notion of a strict supersolution. It is known in the literature (see e.g. [11] for $C^{1}$ sub- and supersolutions); here however we relax the regularity assumptions.

Definition 2.8. We shall say that a supersolution $v$ is a strict supersolution of (1.1) if for any test function $\varphi \in C^{1}$ such that $v-\varphi$ has a minimum at $\left(t_{0}, x_{0}\right)$, then

$$
\varphi_{t}\left(t_{0}, x_{0}\right)+H^{*}\left(t_{0}, x_{0}, v\left(t_{0}, x_{0}\right), \varphi_{x}\left(t_{0}, x_{0}\right)\right)>0 .
$$

We define a strict subsolution of (1.1) in a similar way.

We may now state our next observation as follows. 
Proposition 2.9. Let us suppose that the assumptions (R1) and (H1)-(H7) hold. If $v$ is a supersolution of (1.1), then so is $v+\epsilon$ for any positive $\epsilon$. Moreover, $v+\epsilon$ is a strict supersolution.

Proof. Since $v$ is a supersolution, the inequality in Definition 2.8 is obvious due to the strict monotonicity of $H^{*}$ shown in Corollary 2.4 .

Let us now define the regularized Hamiltonian. For $\delta>0$ we set

$$
\begin{aligned}
& H^{\delta}(t, x, u, p) \\
& = \begin{cases}H(t, x, u, p) & |x| \geq r_{0}(t)+\delta, \\
\left(1-\frac{\lambda}{\delta}\right) H\left(t, r^{*}, u, p\right)+\frac{\lambda}{\delta} H\left(t, r_{0}+\delta, u, p\right) & |x|=r_{0}(t)+\lambda, \lambda \in(0, \delta), \\
H\left(t, r^{*}(t), u, p\right) & |x| \leq r_{0}(t) .\end{cases}
\end{aligned}
$$

Note that $\lambda$ depends on $x$ and $t$. Here is our first observation on $H^{\delta}$.

Lemma 2.10. If $u$ is a subsolution to (1.1), then it is also a subsolution to

$$
d_{t}+H^{\delta}\left(t, x, d, d_{x}\right)=0 .
$$

Proof. The claim follows immediately from the inequality

$$
H^{\delta}(t, x, u, p) \leq H_{*}(t, x, u, p) .
$$

We are ready for a definition of a shifted supersolution $v^{\delta}$. We set

$$
v^{\delta}(t, x)= \begin{cases}v(t, x-\delta) & \text { for } x>\delta \\ v(t, 0) & \text { for } x \in[-\delta, \delta], \\ v(t, x+\delta) & \text { for } x<-\delta\end{cases}
$$

As a result $v^{\delta}$ is a uniformly continuous function.

We have to show that for a given $\epsilon$, for a sufficiently small $\delta$, the function $v^{\delta}+\epsilon$ is indeed a supersolution to (2.4).

Lemma 2.11. Let us suppose that assumptions (R1) and (H1)-(H7) hold and that:

(a) $w$ is piecewise a $C^{1}$ function;

(b) $w$ is a supersolution of (1.1);

(c) $w$ is a supersolution at infinity of (1.1).

Then, for any $\epsilon>0$ there is such a $\delta_{0}(\epsilon)>0$ that for any $\delta \in\left(0, \delta_{0}(\epsilon)\right)$, the function $w^{\delta}+\epsilon$ is a supersolution of (2.4).

Proof. By Proposition 2.9 $w+\epsilon, \epsilon>0$, is a strict supersolution of (1.1). We claim that the restrictions imposed on the behavior of $w$ at infinity permit us to show a stronger inequality than that postulated by Definition 2.8. Namely, there exists $\eta>0$ such that for any test function $\varphi$ such that the difference $w-\varphi$ attains its minimum at $(t, x) \in(0, T) \times \mathbb{R}$ we have

$$
\varphi_{t}(t, x)+H^{*}\left(t, x, w(t, x)+\epsilon, \varphi_{x}(t, x)\right) \geq \eta>0 .
$$

Indeed, we noticed that $H^{*}$ satisfies (2.1) with the same $h_{0}$ as $H$. Thus, we deduce

$$
\begin{aligned}
& \varphi_{t}(t, x)+H^{*}\left(t, x, w(t, x)+\epsilon, \varphi_{x}(t, x)\right) \\
& \geq \varphi_{t}(t, x)+H^{*}\left(t, x, w(t, x), \varphi_{x}(t, x)\right)+h_{0} \epsilon \geq h_{0} \epsilon=: \eta>0 .
\end{aligned}
$$

In other words, (2.5) holds for all $(t, x) \in(0, T) \times \mathbb{R}$, as desired. 
We notice that due to (2.1) the Hamiltonian at infinity $H^{\infty}$ is also strictly increasing with respect to $u$. It is sufficient to pass to the limit in (2.1) to deduce that

$$
H^{\infty}\left(t, u_{2}\right)-H^{\infty}\left(t, u_{1}\right) \geq h_{0}\left(u_{2}-u_{1}\right) .
$$

This inequality combined with (2.3) shows that $w+\epsilon$ is a strict supersolution at infinity.

We will now show that $w^{\delta}+\epsilon$ is a supersolution. We need to show that for a test function such that $w^{\delta}-\varphi$ attains its minimum at $(t, x)$,

$$
\varphi_{t}+H^{\delta}\left(t, x, w^{\delta}+\epsilon, \varphi_{x}\right) \geq 0 .
$$

We first consider $x>r_{0}(t)+\delta$; then we have

$\varphi_{t}(t, x)+H\left(t, x, w^{\delta}(t, x)+\epsilon, \varphi_{x}(t, x)\right)=\varphi_{t}(t, x)+H\left(t, x, w(t, x-\delta)+\epsilon, \varphi_{x}(t, x)\right)$.

We write $y=x-\delta$; hence $y+\delta>r_{0}(t)+\delta$. We notice that $H$ is locally uniformly continuous in $G \times \mathbb{R}^{2}$. Indeed, because of the assumed uniform convergence of $H$ to $H^{\infty}$ for a given $\eta$ we can find such an $R$ where for $|y|,|z| \geq R \geq \mu_{0}$ we have $|H(t, y, w, p)-H(t, z, w, p)|<\eta$. Due to compactness of the set $G \cap B_{R}(0)$, function $H$ is uniformly continuous on $\mathcal{F}=\left(G \cap B_{R}(0)\right) \times\left[-\|w\|_{\infty}-1,\|w\|_{\infty}+\right.$ $1] \times\left[-\left\|w_{x}\right\|_{\infty},\left\|w_{x}\right\|_{\infty}\right]$.

Let us now introduce a new test function by the formula $\psi(t, y)=\varphi(t, y+\delta)$. We have to check that $\left|\psi_{x}\right| \leq\left\|w_{x}\right\|_{\infty}$. This is indeed so because the inequality $(w(t, z)-\psi(t, z)) \geq 0$ for $z \neq y$ in a neighborhood of $y$ implies that $w_{x}^{+}(t, y) \geq$ $\psi_{x}(t, y)$ and $w_{x}^{-}(t, y) \leq \psi_{x}(t, y)$. Since (2.5) holds, we can find $\delta$ so that

$\psi_{t}(t, y)+H\left(t, y+\delta, w(t, y)+\epsilon, \psi_{x}\right) \geq \psi_{t}(t, y)+H\left(t, y, w(t, y)+\epsilon, \psi_{x}\right)-\eta \geq \eta-\eta=0$.

This proves the claim for $x>r_{0}(t)+\delta$.

Suppose now that $\delta<x<r_{0}$. In this case $H(t, x-\delta, u, p)=H\left(t, r^{*}(t), u, p\right)$, and it is by definition smaller than $H^{\delta}(t, x, u, p)$. Then, after setting $y=x-\delta$ and introducing the same new test function $\psi$, by (2.5) we have

$\psi_{t}(t, y)+H^{\delta}\left(t, y+\delta, w(t, y)+\epsilon, \psi_{x}(t, y)\right) \geq \psi_{t}(t, y)+H\left(t, r^{*}, w(t, y), \psi_{x}(t, y)\right) \geq 0$

Our claim holds again.

Now we take $x \in\left[r_{0}(t), r_{0}(t)+\delta\right)$ and proceed as before. We notice that

$$
\begin{aligned}
\varphi_{t}(t, & x)+H^{\delta}\left(t, x, w^{\delta}(t, x)+\epsilon, \varphi_{x}(t, x)\right) \\
= & \varphi_{t}(t, x)+H^{\delta}\left(t, x, w(t, x-\delta)+\epsilon, \varphi_{x}(t, x)\right) \\
> & \psi_{t}(t, y)+H^{\delta}\left(t, y+\delta, w(t, y), \psi_{x}(t, y)\right) \\
& \geq \psi_{t}(t, y)+H\left(t, r^{*}(t), w(t, y), \psi_{x}(t, y)\right) \geq 0 .
\end{aligned}
$$

Let us now suppose that $|x| \leq \delta$. In this case, we denote the modulus of continuity of $H_{*}$ on the closure of $(([0, T] \times \mathbb{R}) \backslash G) \times \mathbb{R}^{2}$ by $\Omega_{H}$ and the modulus of continuity of $w$ by $\Omega_{w}$. After this preparation we see that

$$
\begin{aligned}
\varphi_{t}(t, x) & +H^{\delta}\left(t, x, w^{\delta}(t, x)+\epsilon, \varphi_{x}(t, x)\right)=\varphi_{t}(t, x)+H^{\delta}\left(t, x, v(t, 0)+\epsilon, \varphi_{x}(t, x)\right) \\
& \geq \varphi_{t}(t, x)+H^{\delta}\left(t, x, w(t, x)+\epsilon, \varphi_{x}(t, x)\right)-\Omega_{H}\left(\Omega_{w}(\delta)\right) \\
& \geq \varphi_{t}(t, x)+H^{\delta}\left(t, x, w(t, x), \varphi_{x}(t, x)\right)-\Omega_{H}\left(\Omega_{w}(\delta)\right)+h_{0} \epsilon \geq 0 .
\end{aligned}
$$

The last inequality is the definition of $\delta_{0}(\epsilon)$, which must be so small that $\Omega_{H}\left(\Omega_{w}(\delta)\right)$ $\leq h_{0} \epsilon$. 
If $-r_{0} \leq x<-\delta$ we proceed as follows:

$$
\begin{aligned}
\varphi_{t}(t, x) & +H^{\delta}\left(t, x, w^{\delta}(t, x)+\epsilon, \varphi_{x}(t, x)\right) \\
& =\varphi_{t}(t, x)+H^{\delta}\left(t, x, w(x+\delta)+\epsilon, \varphi_{x}(t, x)\right) \\
& =\psi_{t}(t, y)+H^{\delta}\left(t, y-\delta, w(t, y)+\epsilon, \psi_{x}(t, y)\right) \\
& >\psi_{t}(t, y)+H\left(t, r^{*}(t), w(t, y), \psi_{x}(t, y)\right) \geq 0 .
\end{aligned}
$$

Our claim holds again.

For the remaining cases of negative $x$, i.e. when $x \in\left(-r_{0}(t)-\delta,-r_{0}(t)\right)$, the reasoning is the same. With the previous notation we have

$$
\begin{aligned}
\varphi_{t}(t, x) & +H^{\delta}\left(t, x, w^{\delta}(t, x)+\epsilon, \varphi_{x}(t, x)\right) \\
& =\varphi_{t}(t, x)+H^{\delta}\left(t, x, w(x+\delta)+\epsilon, \varphi_{x}(t, x)\right) \\
& =\psi_{t}(t, y)+H^{\delta}\left(t, y-\delta, w(t, y)+\epsilon, \psi_{x}(t, y)\right) \\
& >\psi_{t}(t, y)+H\left(t, r^{*}(t), w(t, y), \psi_{x}(t, y)\right) \geq 0 .
\end{aligned}
$$

The case of $x<-r_{0}(t)-\delta$ is handled the same as the situation when $x>r_{0}(t)+\delta$. We omit the details.

Finally, we consider points $(t, x)=\left(t, \pm\left(r_{0}(t)+\delta\right)\right)$. These are translations of special points of $H$. Let us first consider $(t, x)=\left(t, r_{0}(t)+\delta\right)$. For any test function $\varphi$ we have to show that

$$
\begin{aligned}
L & :=\varphi_{t}+H^{\delta}\left(t, r_{0}(t)+\delta, w^{\delta}\left(r_{0}(t)+\delta, t\right)+\epsilon, \varphi_{x}\right) \\
& =\varphi_{t}+H^{*}\left(t, r_{0}(t)+\delta, w\left(r_{0}(t), t\right), \varphi_{x}\right) \geq 0 .
\end{aligned}
$$

Since $H^{*}$ is uniformly continuous in $(G \cap([0, T] \times[0, R])) \times[-R, R]^{2}$, we can find $\delta(\epsilon)$ so that (2.5) implies

$$
\begin{aligned}
L & \geq \psi_{t}\left(t, r_{0}(t)\right)+H^{*}\left(t, r_{0}(t), w\left(t, r_{0}(t)\right)+\epsilon, \psi_{x}\left(t, r_{0}(t)\right),\right)-\eta \\
& \geq \eta-\eta=0 .
\end{aligned}
$$

We recall that $H^{*}\left(t, r_{0}(t), v, p\right)=H\left(t, r_{0}(t)^{+}, v, p\right)$.

The point $(t, x)=\left(t,-r_{0}(t)-\delta\right)$ is handled in the same manner. Our claim follows.

Remark 2.12. The assumption that $v+\epsilon$ is a strict supersolution at infinity is technical only for the sake of dealing with unbounded domain.

We are now ready for the proof of our main result. Let us suppose that a subsolution $u$ and a supersolution $v$ satisfy the assumptions. By Proposition 2.9. for any positive $\epsilon, v+\epsilon$ is a strict supersolution; moreover $v+\epsilon$ is a strict supersolution at infinity. Since $v+\epsilon$ is uniformly continuous over $\mathbb{R}$, there is a $\delta_{0}(\epsilon)$ such that for all $\delta \in\left(0, \delta_{0}(\epsilon)\right)$ we have $v^{\delta}(0, x)+\epsilon>u(0, x)$. By Lemma 2.10, $u$ is a subsolution to (2.4), while due to Lemma $2.11 v^{\delta}+\epsilon$ is a supersolution to the same equation, possibly for a smaller $\delta$. Since $H^{\delta}$ is strictly increasing with respect to $u$, we may apply the classical comparison principle to conclude that

$$
u(t, x) \leq v^{\delta}(t, x)+\epsilon
$$

for all $t \in[0, T]$. Since $\delta_{0}(\epsilon)$ goes to zero when $\epsilon \rightarrow 0$ and $v^{\delta}+\epsilon$ converges to $v$, we conclude that

as desired.

$$
u(t, x) \leq v(t, x) \text { for all } t \in[0, T],
$$


We notice that our comparison principle improves the uniqueness result of 8 , Theorem 1.1], because there we assumed an unnecessary structure of our superand subsolutions. Here they are removed, but we restrict the behavior of superand subsolutions at infinity. However, the complete analysis of this issue will be presented elsewhere.

It would be natural to ask the question whether we can repeat the argument when the supersolution is just bounded uniformly continuous and the subsolution is piecewise $C^{1}$ and in addition is a properly defined subsolution at infinity. Unfortunately, there is no obvious answer to this question in sight. Roughly speaking, the flat part of $H$ plays a special role so that we cannot freely trade properties of supersolutions for properties of subsolutions.

\section{ACKNowledgments}

The first and third authors acknowledge stimulating conversations with Professor H. Ishii on the subject of this paper.

The authors thank the referee for constructive remarks.

\section{REFERENCES}

1. G. Barles, B. Perthame, Discontinuous solutions of deterministic optimal stopping time problems, RAIRO Modél. Math. Anal. Numér., 21 (1987), 557-579. MR921827(88k:49032)

2. F. Camilli, A. Siconofli, Time-dependent measurable Hamilton-Jacobi equations, Comm. Partial Differential Equations, 30 (2005), 813-847. MR2153516 (2006c:35034)

3. X.-F. Chen, B. Hu, Viscosity solutions of discontinuous Hamilton-Jacobi equations, Interfaces Free Bound., 10 (2008), 339-359. MR2453135 (2009i:35040)

4. M. Coclite, N. Risebro, Viscosity solutions of Hamilton-Jacobi equations with discontinuous coefficients, J. Hyperbolic Differ. Equ., 4 (2007), 771-795. MR2374224 (2008k:49066)

5. K. Deckelnik, Ch. Elliott, Uniqueness and error analysis for Hamilton-Jacobi equations with discontinuities, Interfaces Free Bound., 6 (2004), 329-349. MR2095336 (2005h:35035)

6. C. De Zan, P. Soravia, Cauchy problems for noncoercive Hamilton-Jacobi-Isaacs equations with discontinuous coefficients, preprint.

7. Y. Giga, Surface evolution equations. A level set approach, Monographs in Mathematics, 99. Basel: Birkhäuser, 2006. MR2238463 (2007j:53071)

8. Y. Giga, P. Górka, P. Rybka, Nonlocal spatially inhomogeneous Hamilton-Jacobi equation with unusual free boundary, Discrete Contin. Dyn. Syst., 26 (2010), 493-519. MR2556496

9. H. Ishii, Hamilton-Jacobi equations with discontinuous Hamiltonians on arbitrary open sets, Bull. Fac. Sci. Engrg. Chuo Univ., 28 (1985), 33-77. MR845397 (87k:35055)

10. Th. Stromberg, On viscosity solutions of irregular Hamilton-Jacobi equations, Arch. Math. (Basel), 81 (2003), 678-688. MR2029244 (2004m:35039)

11. A. Tourin, A comparison theorem for a piecewise Lipschitz continuous Hamiltonian and application to shape-from-shading problems, Numer. Math., 62 (1992), 75-85. MR.1159046 (93d:65064)

Graduate School of Mathematical Sciences, University of Tokyo, Komaba 3-8-1, TOKYO 153-8914, JAPAN

E-mail address: labgiga@ms.u-tokyo.ac.jp

Instituto de Matemática y Física, Universidad de Talca, Casilla 747, Talca, Chile - And - Department of Mathematics and Information Sciences, Warsaw University of Technology, Pl. Politechniki 1, 00-661 Warsaw, Poland

E-mail address: pgorka@mini.pw.edu.pl

Institute of Applied Mathematics and Mechanics, Warsaw University, L. Banacha 2, 07-097 Warsaw, Poland

E-mail address: rybka@mimuw.edu.pl 